\title{
Schema Architectures and Their Relationship to Transaction Processing in Distributed Database Systems
}

\author{
PETER APERS \\ Department of Computer Science, University of Twente, The Netherlands
}

and

PETER SCHEUERMANN

Department of Electrical Engineering and Computer Science, Northwestern Unicersity, Evanston, Illinois 60201

Communicated by Ahmed K. Elmagarmid

\begin{abstract}
We discuss the different types of schema architectures which could be supported by distributed database systems, making a clear distinction between logical, physical, and federated distribution. We elaborate on the additional mapping information required in architecture based on logical distribution in order to support retrieval as well as update operations. We illustrate the problems in schema integration and data integration in multidatabase systems and discuss their impact on query processing. Finally, we discuss different issues relevant to the cooperation (or noncooperation) of local database systems in a heterogeneous multidatabase system and their relationship to the schema architecture and transaction processing.
\end{abstract}

\section{INTRODUCTION}

The database architectural framework proposed by ANSI/SPARC [26] was developed in order to support evolution and changes in the use of the data as well in their management and internal representation. This architecture is based on three levels of data description, the central and most important one being the conceptual schema (see Figure 1). The conceptual schema describes all the entities, their relationships, and the integrity constraints relevant to an organization's real world which should be maintained in the database. The 


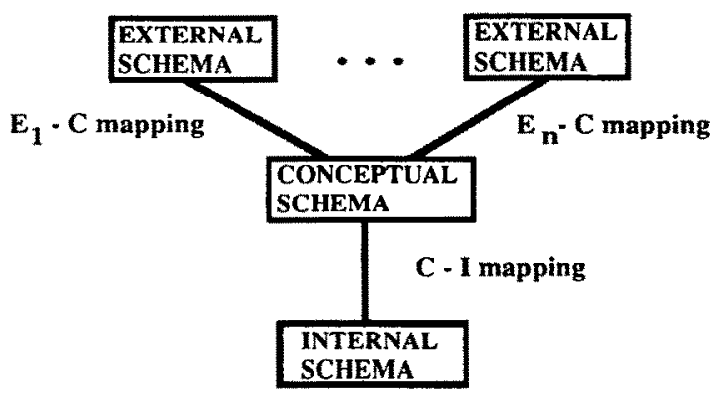

Fig. 1. ANSI/SPARC architecture.

external schemas, denoted by $E_{1}$ to $E_{n}$ in Figure 1, describe parts of the information in the conceptual schema as they should be seen by different user groups. The conceptual and external schema descriptions may use different data models (e.g. relational, network, or semantic data models). Furthermore the external schemas are limited, not only in the sense that they can contain only a subset of the information described in the conceptional schema, but also by the fact that they cannot incorporate additional intcgrity constraints. The third schema type in the proposed architecture, the internal schema (denoted by $I$ in Figure 1), describes how the information contained in the conceptual schema is physically stored in terms of access paths, encodings, etc.

In addition to the three schemas represented by boxes in Figure 1, the ANSI/SPARC architecture also contains a number of mappings, represented by edges in the diagram. The external-conceptual mappings show how the information contained in the given user views is related to the information described in the conceptual schema. Similarly, the conceptual-internal mapping describes the correspondence between the logical items of information and their counterparts in physical storage. The conceptual schema is stable over time, compared to the external and internal schemas. However, if a new entity or attribute must be added to the conceptual schema, this does not affect the external schemas, but only the $E-C$ mappings, ensuring logical data independence. Similarly, if, for example, some access paths are added to the internal schema, the conceptual schema is not affected, but only the I-C mapping, guaranteeing physical data independence.

More recently an additional dimension to the description of data has been identified, the intension-extension dimension [16]. This dimension is important for the description of metadata. However, as this dimension is orthogonal to the ANSI/SPARC schema dimension, we are not concerned with it here. Our concerns are the extensions to the ANSI/SPARC schema architecture for 
distributed systems and their relationship to transaction processing, in particular query processing and concurrency control.

While different extensions to the above-discussed schema architecture have been proposed for distributed databases [1, 11], the concepts have not been clearly defined; very often the same name is used to refer to descriptions at different levels of abstraction. In this paper we define the different types of schema architecture which could be supported in distributed database systems and make a clear distinction between logical distribution and physical distribution. We further discuss the additional mapping information required in architectures based on logical distribution to support not only retrieval operations, but also updates and update propagation.

Aspects of the relationship between schema architecture in distributed database systems and various components of transaction processing have been examined in various contexts. The experience with Multibase [12] has shown that logical distribution implies that even simple queries may require expensive processing. The fact that the overall schema architecture may not be visible to every local database system implies that query optimization and concurrency control can be performed in more or less limited form, depending upon the level of cooperation between the systems. We discuss restrictions to the schema architecture for the case when the systems are noncooperative, as well as the additional mapping information necessary in the schema to specify various types of cooperation.

In this paper, we present a tutorial view of distributed database systems and multidatabase systems which is based on the classification of schema architectures and the type of communication available among the participating systems. The paper is organized as follows. In Section 2 we discuss the basic distribution strategies and the corresponding schema architectures for distributed database systems. Section 3 addresses itself to the impact of logical distribution on query processing and to the relationship between the mapping description in logically distributed systems and object-based specification. In Section 4 we discuss the different issues relevant to the cooperation or noncooperation of local database systems in distributed database systems and the effect of these issues on the schema architecture and transaction processing.

\section{SCHEMA ARCHITECTURE TYPES FOR DISTRIBUTED DATABASES}

We now shall investigate the basic frameworks for schema architecture which can be supported by distributed database systems. In the process we shall distinguish between "traditional" distributed database systems [24, 29] 
and multidatabase systems $[13,15]$. The three basic strategies for distribution which we identify are physical, logical, and federal distribution.

\subsection{PHYSICAL DISTRIBUTION}

This type of distribution is encountered in a top-down system design [1] in which the conceptual schema is designed the same way as in a centralized system and then decisions are made about where to store the data on different sites in order to increase efficiency of access and improve data availability. Physical distribution can be achieved through data partitioning, where the logical data objects are divided into fragments which are stored at one site, or through replication, where the logical objects (or fragments of them) are stored at multiple sites. In [4] we presented some algorithms to determine the fragments of logical objects and their locations.

Figure 2 illustrates the schema architectures for a system whose data are physically distributed among two locations. We now have a hierarchy of internal schemas: $I_{1}$ describes the distribution, while $I_{2}$ and $I_{3}$ describe how the data are stored and the available access paths for single computers, just as in the ANSI/SPARC architectures. Each physical location may have a complete copy of the distributed database system, or alternatively, each node may only be equipped with a storage and retrieval system while the transaction management is monitored by one node $[5,29]$. In either case, as far as the external users are concerned, only one conceptual schema is seen.

With regard to the mappings, only the $C-I_{1}$ mapping is different from the $C$-I mapping appearing in the original framework. Let us consider, using an entity-relationship (E-R) diagram [21], the simple conceptual schema illustrated in Figure 3. The rectangular boxes stand for entity sets (EMP and DEPT), while the diamond-shaped boxes represent relationship sets. Using a semifor-

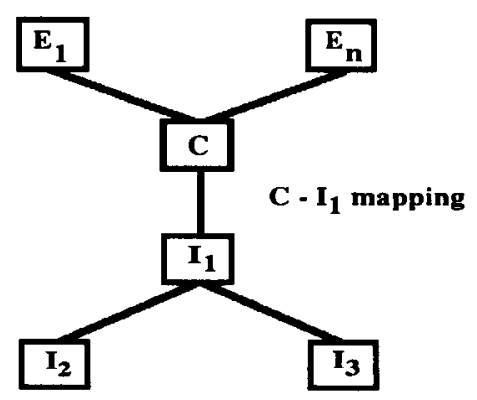

Fig. 2. Physical distribution architecture. 


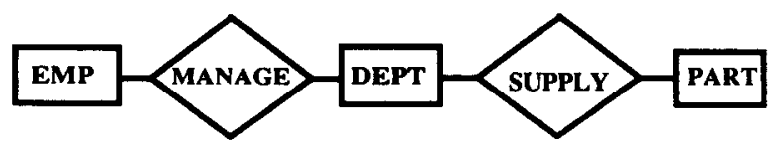

Fig. 3. E-R conceptional schema.

mal notation, we may convey a possible distribution through the following $C-I_{1}$ mapping:

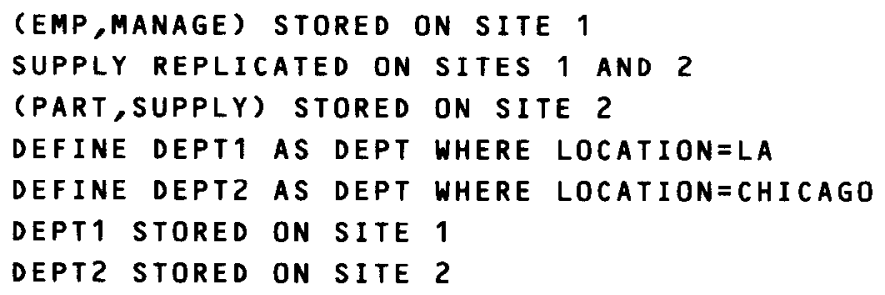

\subsection{LOGICAL DISTRIBUTION}

The logical distribution strategy is usually associated with the bottom-up approach to database system design. In this approach a number of preexisting, possibly heterogeneous databases residing at the same or different locations are interconnected to present a logically integrated view by means of a global conceptual schema $[13,25]$. The problems involved in integrating preexisting databases are numerous: solving inconsistencies in data, structural differences, differences in level of abstraction, etc. [6]. Due to these problems, it has been suggested [15] that instead of designing a single global conceptional schema, it may be easier to provide the users with a collection of conceptual schemas that integrate subsets of the existing databases. However, as far as the schema architecture is concerned, it does not make any difference whether the global conceptual schema consists of one part or of multiple parts. The schema architecture that we obtain for logical distribution is illustrated in Figure 4.

The global conceptual schema (GC) provides the global view through which the users of the integrated system can obtain access via the global external views, denoted by $\mathrm{GE}_{1}$ through $\mathrm{GE}_{m}$ in Figure 4 . Observe that since the preexisting database systems with conceptual schemas $C_{1}$ through $C_{n}$ can continue to operate autonomously, we may also have local external schemas, such as $\mathrm{LE}_{11}, \ldots, \mathrm{LE}_{1 k}$, which have no knowledge of the integrated view.

Logical and physical distribution constitute two basic strategies, based on which we can identify two types of distributed database systems. "Traditional" distributed database systems use the physical distribution approach, while 


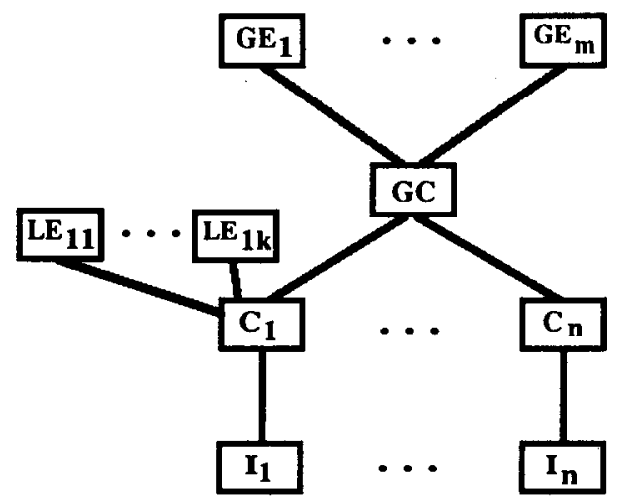

Fig. 4. Logical distribution architecture.

those systems using logical distribution are becoming more frequently referred to as multibase [13] or multidatabase systems [15].

The mappings from the global conceptual schema GC to the underlying conceptual schemas $C_{1}$ through $C_{n}$ must describe how the entities and relationships in GC relate to the entities and relationships of $C_{1}$ through $C_{n}$. For retrieval only, this can be accomplished via a view derivation facility, such as the one described in [6], where the rules for obtaining the logical data objects of the global conceptual schema from the underlying conceptual schemas are expressed in the query language of the multidatabase system.

In addition to the retrieval information, the mapping discussed above must also describe how updates performed at a higher level of abstraction (the GC schema) are to be supported in terms of operations at lower levels of abstraction. Currently the existing prototype multidatabase systems [13, 15, 25] do not support updates at the global level-this capability is obviously needed if such systems are to become fully operational.

Some of the additional complexity in logical distribution versus physical distribution is due to the facts that (1) the global conceptual schema may impose its own integrity constraints (not present at the lower conceptual levels) and (2) the underlying conceptual schemas may overlap in information.

We shall illustrate some of these problems by doing a variation on the E-R diagram from Figure 3. Let us assume that two independently designed databases, represented by the conceptual schemas $C_{1}$ and $C_{2}$ in Figure 5 , are to be integrated. The instances in DEPT ${ }_{1}$ and DEPT 2 may be overlapping, while all other entities and relationships sets in $C_{1}$ and $C_{2}$ are mutually exclusive. In the global conceptual schema GC we want to "see" all entities and relation- 


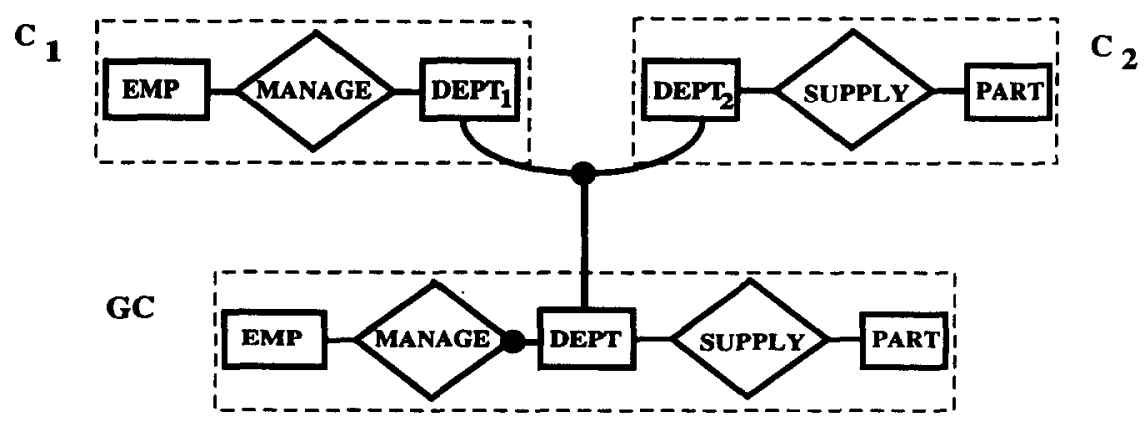

Fig. 5. Global conceptual schema with ovcrlapping elements.

ships types from $C_{1}$ and $C_{2}$, but in addition we impose the constraint that the relationship set MANAGE is to be total [21] on the entity set DEPT, in other words, that every department in GC must have a manager (see Figure 5). In our diagrammatic representation we represent a total relationship by drawing a dot on the edge of the relationship connected to the total entity set. This constraint in fact restricts the instances of DEPT to be a subset of DEPT ${ }_{1}$, namely DEPT = (DEPT1 and DEPT2) IN MANAGE = DEPT 1 IN MAN$A G E$. A set theoretic definition of the legal DEPT instances under this constraint would be

$$
\operatorname{DEPT}=\left\{d \mid d \in \operatorname{DEPT}_{1} \wedge \exists m \in \text { MANAGE s.t. } m[\text { dept.id }]=d[\text { dept.id }]\right\}
$$

One strategy for allowing updates at the global conceptual level could be to choose an interpretation that minimizes the "interference" with the underlying databases. In this case, if a department instance is to be deleted from DEPT, the mapping description would be

$$
\begin{aligned}
& \text { DELETE } d \in \text { DEPT } \Rightarrow \text { THEN DELETE } d \in \text { DEPT }_{1} ; \\
& \text { If } \exists m \in \text { MANAGE s.t. } m \text { [dept.id] }=d \text { [dept.id] THEN DELETE } m
\end{aligned}
$$

Although the global conceptual schema GC consists of $C_{1}$ and $C_{2}$, in this case a delete in DEPT is to be interpreted only as a delete in $C_{1}$, with the delete of $d$ in DEPT propagated also to MANAGE (a relationship can exist only if the related entities exist).

Let us assume now that the databases $C_{1}$ and $C_{2}$ are integrated so that no additional constraints are incorporated in GC, i.e., both relationships MANAGF. and sUPPLY are partial. This corresponds to a definition DEPT $=\mathrm{DEPT}_{1} \cup \mathrm{DEPT}_{2}$. 
A delete operation from DEPT is to be interpreted now as

DELETE $d \in$ DEPT $\left.\Rightarrow\left\{\begin{array}{l}\text { DELETE } d \in \text { DEPT }_{1} ; \\ \text { If } \exists m \in \text { MANAGE s.t. } \\ m[\text { dept } . \text { id] }=d \text { [dept.id] }] \\ \text { THEN DELETE } m\end{array}\right\} \| \begin{array}{l}\text { DELETE } d \in \text { DEPT }_{2} ; \\ \text { If } \exists s \in \text { SUPPLY s.t. } \\ s[\text { dept.id] }=d \text { [dept.id] }] \\ \text { THEN DELETE } s\end{array}\right\}$.

Since the same object $d$ could be present in both databases, the two instances of $d$ must be deleted followed by the appropriate update propagation. The operator $\|$ specifies that the two sequences of operations enclosed in braces could be performed in parallel.

\subsection{FEDERAL DISTRIBUTION}

The next distribution approach, which we call federal distribution, combines the basic features of logical and physical distribution. The term federal architecture was introduced in [11], but the ideas behind it have been used earlier by a number of systems, such as SYSTEM $\mathrm{R}^{*}$ [29].

The basic schema architecture for a federally distributed system with two components is illustrated in Figure 6. The conceptual schema of each component, i.e., database system, consists of two parts. One part corresponds to its own data (e.g. $C_{11}$ and $C_{21}$ ), physically stored in the system as described by the corresponding internal schema. The second part corresponds to shared data (e.g. $C_{12}$ and $C_{22}$ )-data owned by a different database system which makes them visible to the first one through its conceptual schema.

To illustrate this type of architecture let us consider again the two conceptual schemas $C_{1}$ and $C_{2}$ from Figure 5. Instead of integrating them into a global conceptual schema as before, we opt for allowing each system to see the DEPT information of the other system, which augments the schemas as shown in Figure 7.

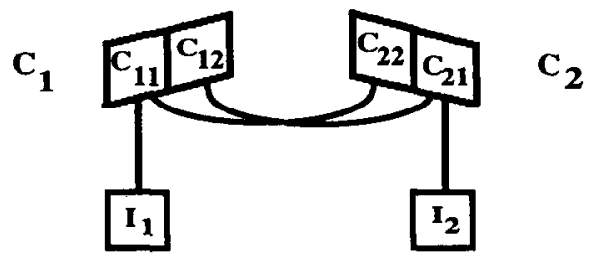

Fig. 6. Federal distribution architecture. 


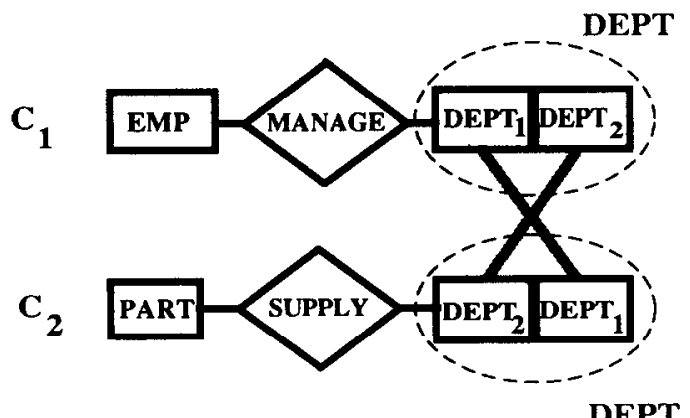

Fig. 7. Federally distributed E-R diagram.

In the more general case a whole collection of conceptual schemas may participate in a federal distribution, with different subsets of the schemas sharing information among their constituents. The SYSTEM $R^{*}$ project [29] was the first to advocate this type of decentralized ownership and autonomy of the databases.

A variant of the federal distribution strategy may be required if one of the constituents is a personal database. A personal database may have access to other global information, but its data may not be shared by other users. In terms of Figure 6 this means that if $C_{1}$ were to describe a personal database, $C_{2}$ would consist only of its own data $\left(C_{21}\right)$, and the mapping $C_{12}-C_{22}$ would also be inhibited.

\subsection{EXPORT SCHEMAS}

As we have seen in the previous subsections, a conceptual schema could be constructed from a number of underlying conceptual schemas. The conceptual schema at the higher level of abstraction may see all the lower-level data objects or only part of them, depending upon how the structure differences are resolved in the process of integration [17]. Some systems explicitly have used another level of schema description, the export schema, to separate issues of incompatible data handling and data integration from homogenization issues caused by the fact that conceptual schemas may use different data models [13].

Figure 8 illustrates the configuration where a number of export schemas are derived from one on top of a conceptual schema. We should observe here that unlike external schemas, which may not incorporate integrity constraints, the export schemas, using a different representation, describe the disjoint 


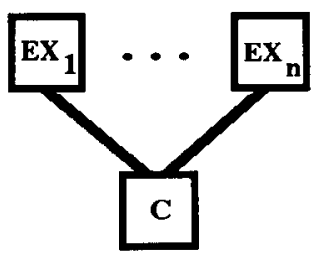

Fig. 8. Export schemas.

parts of a conceptual schema which may be seen at a higher level of abstraction and thus correspondingly may contain integrity constraints.

\subsection{VARLANTS OF LOGICAL DISTRIBUTION}

Another possibility is to have an external schema derived from several conceptual schemas. Figure 9 shows this architecture. The only difference between this and the logical distribution is that no integrity constraints can be specified between the different databases.

This schema architecture is especially useful for giving the user access to several databases without providing a complete integration of them. In that respect, it is closely related to the multidatabase approach without interdatabase integrity constraints.

Another interesting schema architecture is shown in Figure 10. There we have an external schema above two other external schemas. This type of architecture can be used by a company that only has the right of access to different databases via external schemas $E_{2}$ and $E_{3}$ and provides services to users by creating the external schema $E_{1}$. This company has no means to change $E_{2}, E_{3}$, or their respective mappings to the underlying conceptual schemas.

Similar variants can also be obtained for the federal distributions, but we will not discuss these here.

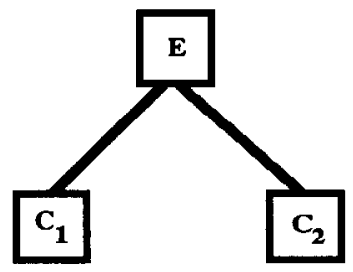

Fig. 9. Logical distribution without integrity constraints. 


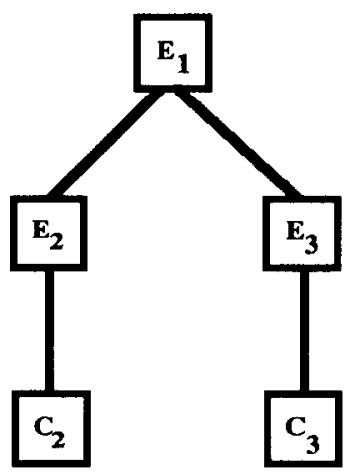

Fig. 10. Integrated access through external schemas.

\section{ISSUES IN LOGICAL INTEGRATION}

Logical integration, sometimes referred to as view integration [6], is a process which is most important for centralized as well as distributed databases. In this section, we shall concentrate on the problems that are specific to logical integration in multidatabase systems which are using a logical distribution approach. The basic schema architecture configurations (for distributed database systems) which we discussed earlier could obviously be combined to obtain a whole hierarchy of conceptual schemas. We shall also discuss in this section the relationship between this architecture approach and object-oriented system design.

\subsection{VIEW INTEGRATION AND QUERY PROCESSING}

Following [6], we distinguish between two types of problems in the integration of views: schema integration and data integration. Schema integration includes the resolution of naming conflicts, scale differences, and structural differences. Naming conflicts are solved by appropriate renaming; scale differences are handled by defining appropriate conversion tables or functions. Structural differences, which constitute the most difficult problems in schema integration, are usually solved by producing the appropriate generalizations of the similar data objects $[6,7,17]$.

Rules for integrating objects in a generalization based on the similarity of the underlying domains and attributes are presented in [7], and in [17] a set of corresponding schema transformations operations is given. We shall restrict ourselves here to an example which illustrates the generalization principle. Let 

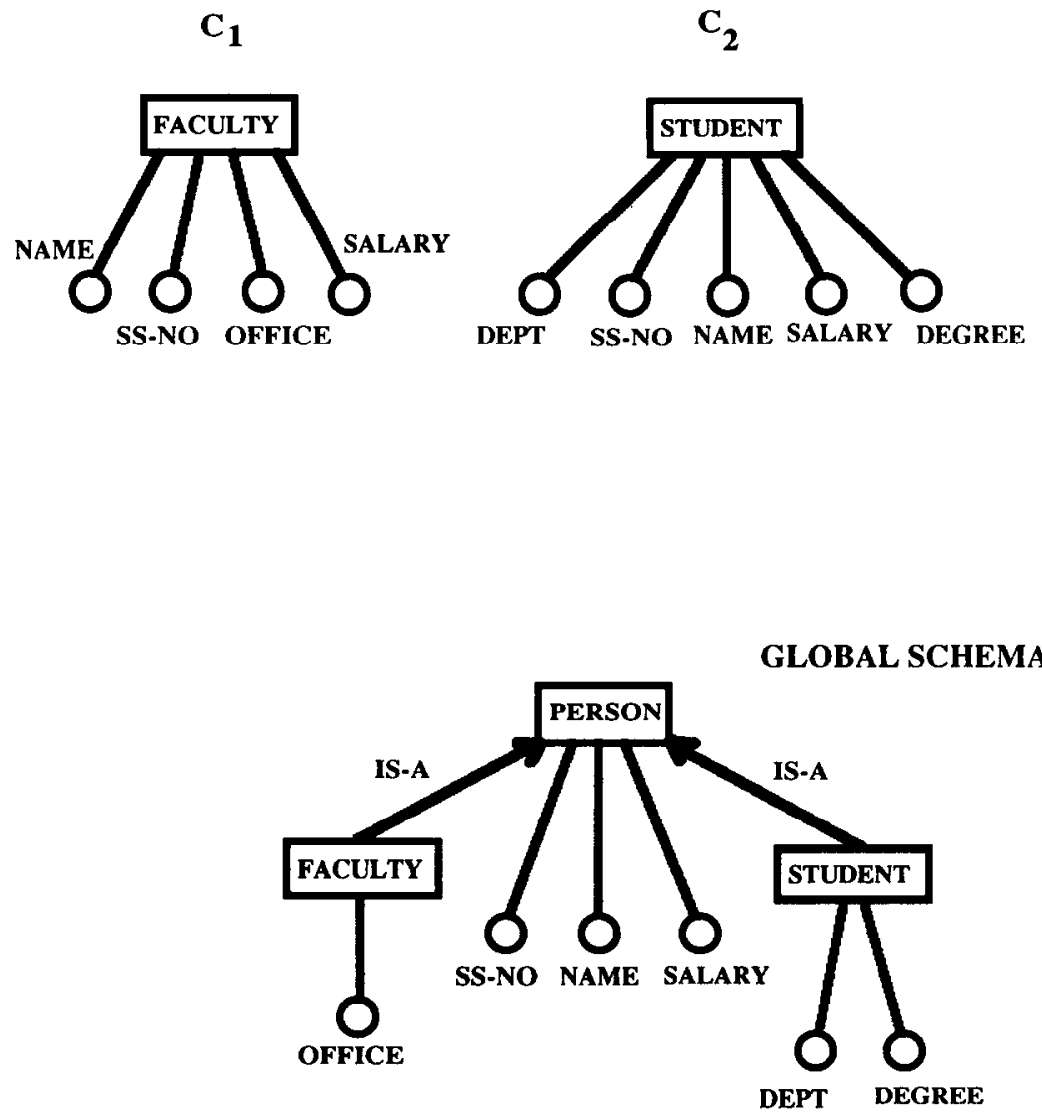

Fig. 11. Integration with MEET generalization.

us assume that we want to integrate the two sample databases of Figure 11(a) representing the FACULTY and STUDENT entity sets. The traditional method of integration would be to create the outerjoin of the FACULTY and STUDENT entity sets, but this introduces unnecessary null values (OFFICE values for STUDENTS who are not FACULTY, and DEPT and DEGREE values for FACULTY who are not STUDENTS); instead, we can integrate the two sets via the generalization hierarchy depicted in Figure 11(b). The generalized entity set PERSON contains the attributes common to FACULTY and STUDENT, i.e. type $($ PERSON $)=$ type (FACULTY $) \cap$ type (STUDENT). The instances in PERSON are the instances in FACULTY or STUDENT, i.e. $\operatorname{dom}($ PERSON) $=\operatorname{dom}$ (FACULTY) $U$ dom(STUDENT). The directed arrows labeled IS-A from FACULTY or STUDENT to PERSON indicate that the two former are subtypes of the last and inherit all its 


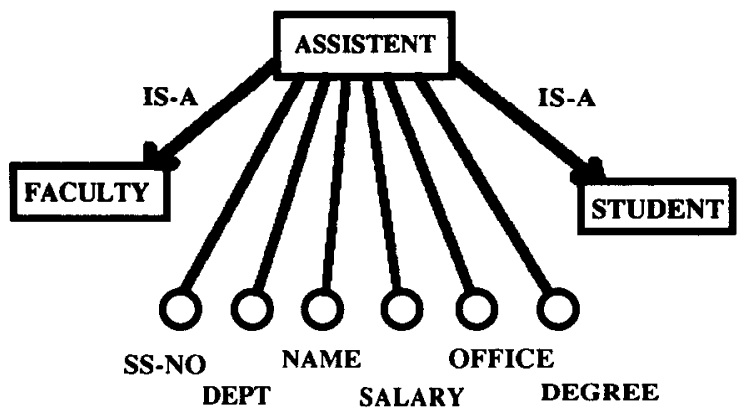

Fig. 12. Join generalization.

attributes.

Two other ways of constructing a global schema from the databases in Figure 11 may be desirable. We may want to see in the global view only the generalized entity set PERSON, i.e., only the common attributes. Alternatively, we may want to see the dual generalized entity set whose type is the union of both types and whose domain is the intersection of both domains. Figure 12 shows the generalized entity set ASsISTANT whose domain includes those instances which are both FACULTY and STUDENT. The generalization depicted in Figure 11 has been named a meet generalization, while the one shown in Figure 12 has been named a join generalization [17].

Once we have decided upon the structure of the global conceptual schema, we still have to solve the problem of data integration, i.e., we must assign appropriate values to the attributes in terms of the values in the underlying schema. Problems occur when the underlying databases overlap and disagree on some attribute values of the same real-life object. Let us refer again to the schema integration represented by Figure 11. NAME is the key on an entity in each local database $\left(C_{1}\right.$ or $\left.C_{2}\right) . C_{1}$ may contain, for example, a value of 40,000 for the salary of ADAM, while $C_{2}$ may contain the value of 8,000 . One possible reason for this inconsistency is that the salary attributes in $C_{1}$ and $C_{2}$ are homonyms, and thus it is legitimate for the same real-life person to have one salary as a faculty member and another as a student (presumably at different institutions).

Multidatabase systems have proposed a number of solutions to solve this type of discrepancy. In [15] the two salary values would be treated as corresponding to different attributes. In addition, as far as the data presentation is concerned, the user would be presented with the answer as a set of sets. Thus, in response to the query: "retrieve all persons with a salary above 10,000 ," the answer will consist of (1) the set of qualifying persons who are only faculty members, (2) the set of qualifying persons who are only students, 
and (3) the set of instances representing persons who are both qualified students and faculty. Thus, in the last set the same person will be represented by two instances, each one with its own salary value. As far as query processing is concerned, the solution strategy proceeds almost the same as in a physically distributed system. The only exception is that the site assembling the final result would have to store the temporary results and merge them to create the different answer sets.

A different approach to data integration was adopted in $[12,6]$. There the inconsistencies were resolved by defining the salary attribute of the generalized entity PERSON as an aggregate function for the overlapping data values. For example, we could define SALARY as

SALARY (PERSON): =

CASE

$\begin{array}{lll}\text { PERSON IN FACULTY - STUDENT: } & \text { SALARY (FACULTY) } \\ \text { PERSON IN STUDENT - FACULTY: } & \text { SALARY (STUDENT) } \\ \text { PERSON IN FACULTYOSTUDENT: } & \text { SALARY (STUDENT) + } \\ & & \text { SALARY (FACULTY) }\end{array}$

ENDCASE

The selection query considered beforc, "retrieve all persons with a salary over 10,000 ," could no longer be processed as two independent subqueries over STUDENT and FACULTY, because we might need to compute the salary of a person as the sum of the two salaries. Processing a selection or projection query in such a multidatabase system can be as expensive as processing a join in a distributed database system using physical distribution.

In processing join queries in traditional distributed database systems the semijoin operation has been recognized as an important tool for reducing the amount of data to be transmitted [3]. A semijoin of a relation $R$ by another relation $S$ selects the tuples in $R$ which can be joined to tuples in $S$. More formally, let us define

$R \bowtie S=$ the natural join of $R$ and $S$ on the common attribute $C$, $R \ltimes S=$ the semijoin of $R$ by $S=\prod_{R}(R \bowtie S)=\{t \mid t \in R \wedge \exists p \in S$ s.t. $t[c]=$ $p[c]]$.

The semiouterjoin operation has been used in [12] in order similarly to reduce the cost of processing queries in multidatabase systems using aggregate functions for overlapping data values. The semiouterjoin of $R$ by $S$ partitions $R$ into two parts: $|R \ltimes S|$, the overlap part of $R$, which corresponds to the semijoin; and $R-|R \ltimes S|$, the complement of the semijoin, called the private part of $R$. We illustrate the effect of the semiouterjoin operations using the materialization of the STUDENT and FACULTY sets shown in Figure 12 (note that some of the attributes have been omitted to save space). 
In performing a selection query on the entity sets $E_{1}, \ldots, E_{n}$, stored at local sites $i(i=1, \ldots n)$, which are subtypes of the generalized type $E$, the obvious strategy would be to have each site send all its tuples in a single move to a central site, which would perform the join and then apply the appropriate aggregate functions. However, in many cases a semiouterjoin strategy consisting of the following sequence of data moves would result in overall cost reduction:

Step 1: Each local site $i$ sends its IDs to a central site

Step 2: The central site computes for each $E_{i}$ the semiouterjoin of $E_{i}$ by the union of the remaining $E_{j}$ 's on the ID attribute. For each $E_{i}$ we obtain at the central site its private part $\operatorname{pr}\left[E_{i}\right]$ and its overlap part ov $\left[E_{i}\right]$.

Step 3: The central site sends to each site $i$

$$
\min \left\{\operatorname{pr}\left[E_{i}\right], \text { ov }\left[E_{i}\right]\right\} .
$$

Step 4: Each site $i$ sends to the central site its overlap part ov $\left[E_{i}\right]$ and the reduced private part, which is the result of performing the local selection.

In our example the reduced private part of FaCULTY, i.e., pr[FaCULTY] [sALARY $>10,000$ ], would contain only the tuples corresponding to BERMAN

FACULTY

\begin{tabular}{|l|c|l|}
\hline NAME & SALARY & OFFICE \\
\hline \hline HARRIS & 30,000 & TECH \\
\hline BERMAN & 40,000 & HUMANITIES \\
\hline CHIRILLO & 5,000 & CONVITO \\
\hline RAMIREZ & 35,000 & HUMANITIES \\
\hline CLARK & 8,000 & FINE ARTS \\
\hline
\end{tabular}

OVERLAP PART (OF FACULTY)

\begin{tabular}{|l|c|l|}
\hline NAME & SALARY & OFFICE \\
\hline \hline CLARK & 8,000 & FINE ARTS \\
\hline HARRIS & 30,000 & TECH \\
\hline
\end{tabular}

STUDENT

\begin{tabular}{|l|c|l|}
\hline NAME & SALARY & DEPT \\
\hline \hline CLARK & 5,000 & EECS \\
\hline LEVY & 7,000 & HISTORY \\
\hline HARRIS & 30,000 & LAW \\
\hline HAJDU & 6,000 & CHEMISTRY \\
\hline
\end{tabular}

SEMIOUTERJOIN

PRIVATE PART (OF FACULTY)

\begin{tabular}{|l|c|l|}
\hline NAME & SALARY & OFFICE \\
\hline \hline CHIRILLO & 5,000 & CONVITO \\
\hline BERMAN & 40,000 & HUMANITIES \\
\hline RAMIREZ & 35,000 & FINE ARTS \\
\hline
\end{tabular}

Fig. 13. Semiouterjoin of FACULTY by STUDENTS (on NAME). 
and RAMIREZ. The complete algorithms for semiouterjoin processing are given in [12].

\subsection{LOGICAL INTEGRATION AND OBIECT-ORIENTED SYSTEM DESIGN}

The conceptual schema must embody a description of the object types, relationships, and constraints to be observed in a database. Furthermore, with regard to constraints this description must encompass static properties, i.e. the permissible database states, as well as dynamic properties, i.e. specifications of how these constraints are to be reinforced. Once we allow for the different distribution strategies in Section 2, the schema architecture could consist of an arbitrary hierarchy of conceptual schemas. As we have seen, it now becomes necessary to describe not only how objects in a schema at a given level of abstraction are composed, but also how operations at the higher level are to be mapped to operations at lower levels. In particular, if update propagation can occur, an individual operation at some level in the schema hierarchy may be translated into one or more transactions at the next lower level in the hierarchy. A similar duality between operations and transactions is also encountered when dealing with nested transactions in [10].

A large number of software specification methodologies have been proposed in the past few years. However, as is the case with the multilevel specification approach of [23], most of these methodologies use different tools

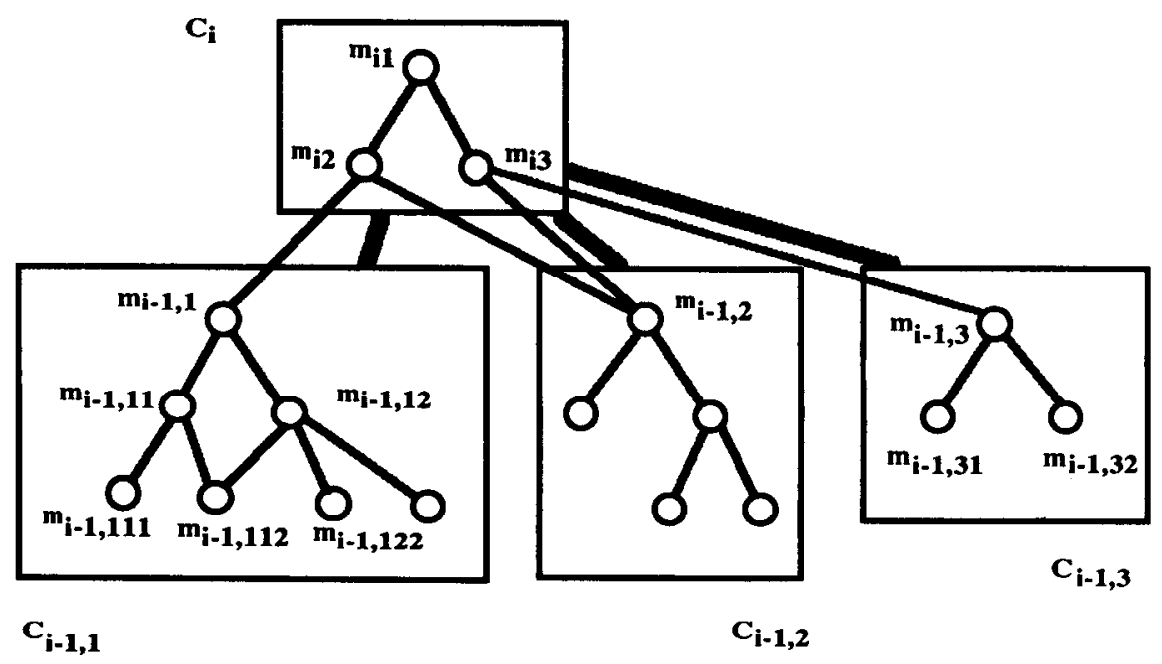

Fig. 14. Module hierarchy in schema with logical distribution. 
for the various phases in the system life cycle which are not entirely compatible. In contrast to these, the object-oriented specification methodology introduced by Weber [27, 28] offers a uniform modeling tool, the module, which has been extended to allow not only for the description of a conceptual schema [21], but also for the description of the database functions and their execution [22].

This object-oriented approach is based on the extended notion of a module. Modules consist of four parts: an export interface, an import interface, a module body, and common parameters [28]. The module body embeds a parametrized abstract data type: it gives the structure of the defined data type in terms of its constituents - the formal parameter data types-and defines the operations on objects of the defined type in terms of operations on the parameter data types. The module concept allows for the formation of a hierarchy of types and operations such that (1) a module is assigned to level $i$ if its structure (type) is defined in terms of subtypes corresponding to modules at level $i-1$, and (2) an operation defined in a module at level $i$ may call subordinates operations in none, some, or all of its component modules at level $i-1$ [28].

We illustrate briefly how this object-oriented specification approach can be applied to the description of the schema architecture in a distributed database system based on logical distribution. Figure 14 describes the module hierarchy for a partial hierarchy of conceptual schemas. In this example the conceptual schema $C_{i}$ has three components at level $i-1: C_{i-1,1}, C_{i-1,2}$, and $C_{i-1,3}$. Each conceptual schema may have its own module hierarchy, as indicated by the corresponding (single-line) edges; the double-line edges, on the other hand, stand for the mappings between $C_{i}$ and $C_{i-1, j}(j=1,2,3)$.

The mappings between $C_{i}$ and $C_{i-1, j}$ must describe how the operations at level $i$ are to be executed in terms of operations at level $i-1$. In particular, given the module hierarchy, within each schema they must describe the "boundary" conditions, i.e. how operations of the leaf nodes (modules) at
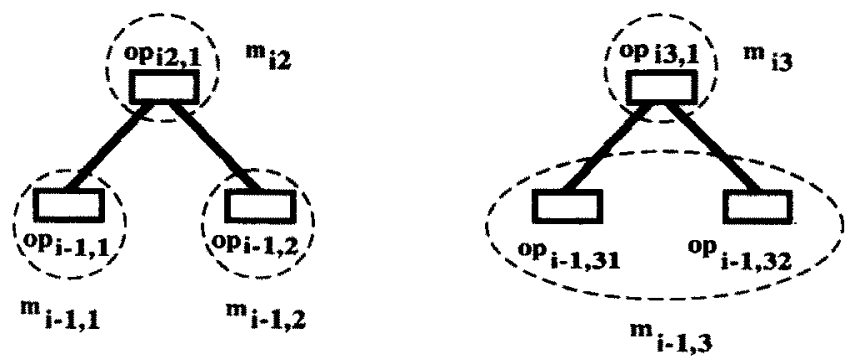

Fig. 15. (Partial) operation hierarchy. 
level $i$ are to be translated into operations of the root nodes (modules) at level $i-1$. Part of the operation hierarchy which corresponds to this mapping specification is shown in Figure 15. The diagram shows which subordinate operations are called and the modules which encapsulate them. In addition, if an operation in the level- $i$ schema is composed of a number of operations in different subschemas at level $i-1$, it is necessary to specify a legal execution schedule [22] in order to allow for concurrent processing.

Finally, as will be discussed in the next section, in order to allow for different degrees of system cooperation the mapping information between the conceptual schemas must be augmented dynamically to include information available in the data directories.

\section{ISSUES IN SCHEMA ARCHITECTURES AND COOPERATION}

In this section we first discuss how a given schema architecture can be mapped on a number of database systems, and in the process we distinguish further between heterogeneous and homogeneous systems and between virtual and physical database systems. We define the notion of cooperativeness among database systems and discuss the implications of cooperativeness on transaction processing.

\subsection{MAPPING THE SCHEMA ARCHITECTURE TO PROCESSORS}

Traditionally, there is a one-to-one correspondence between the three layers of data description and a database system. The conceptual schema, its underlying internal schema, and the external schemas derived from it are known to one database system, which acts according to the information contained in them. However, there is no reason why one database system cannot support an arbitrary hierarchy of conceptual schemas and their related internal and external schemas, or, at the other extreme, why it should not support only a given conceptual schema without its underlying internal schema.

Figure 16 shows how three database systems support an arbitrary schema architecture consisting of five conceptual schemas and three internal schemas (external schemas have been omitted for simplicity). Each database system is indicated by a dashed rectangle which contains the schemas and mappings that are supported by it. In this example, $\mathrm{DS}_{1}$ supports $C_{1}$ and the mappings $C_{1}-C_{2}$ and $C_{1}-C_{3}$. A dashed rectangle may represent a centralized or a distributed database system. If the data described by $I_{1}$ and $I_{2}$ are stored at different sites, then $\mathrm{DS}_{2}$ is a distributed database system. This type of distributed database system consists of identical database systems running at the different sites using one concurrency control mechanism, and will there- 


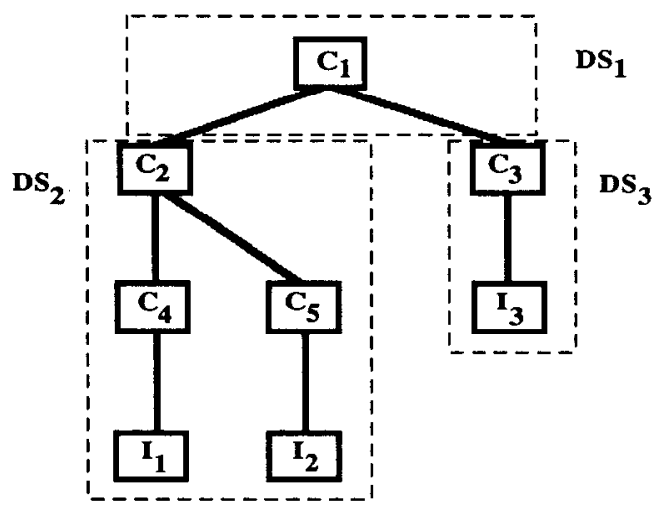

Fig. 16. Arbitrary schema architecture and supporting database systems.

fore be called a homogeneous distributed database system. Homogeneity in this case refers to the database system and not to the data model used. Most of the research on distributed database systems has been concentrated on this type $[14,18,20,24,29]$.

Figure 16 also depicts another type of distribution, which is callcd heterogeneous, because different database systems are used to support the overall schema architecture. Since $\mathrm{DS}_{1}, \mathrm{DS}_{2}$, and $\mathrm{DS}_{3}$ are different database systems, they all have their own query optimizer and concurrency control mechanisms. In a heterogeneous environment, it is important to know what type of communication between the database systems is possible. We classify the communication interface of a database system as follows:

(1) Noncooperative. This describes the situation when a database system can only be accessed via end-user queries. Hence, the only communication among the systems consists of query requests and their corresponding answers. This situation may occur if a database system is developed solely for centralized purposes. Obviously, query optimization and processing and concurrency control become more difficult if a noncooperative database system is used in a heterogeneous distributed database system.

(2) Cooperative. This describes the situation when in addition to communication at the query level, exchange of information contained in the data dictionary and information concerning concurrency control is also possible.

In the next subsection we will elaborate more on the type of information required for query processing and concurrency control in the cooperative case in order to achieve better performance than in the noncooperative case.

Very little research has been done on the issue of noncooperative versus cooperative database systems. MERMAID [25] is a project that deals with the 


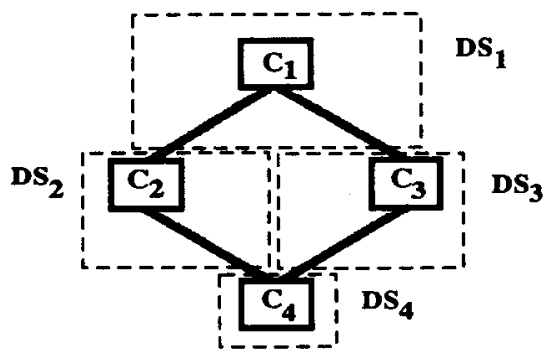

Fig. 17. A cyclic graph schema architecture supported by four database systems.

noncooperativeness of existing database systems. The problem is solved by defining a separate module for each noncooperative database system, which issues queries and receives their answers. Besides these application modules there is a central module, which optimizes queries and delegates the processing of queries to the application modules. The application modules may transmit data to each other in order to process a query. For example, let us assume that $\mathrm{DS}_{2}$ and $\mathrm{DS}_{3}$ in Figure 16 are noncooperative database systems. Then in the MERMAID approach DS 1 consists of the central and the application modules.

We now shall discuss the restrictions on the schema architecture caused by noncooperativeness. In Figure 17 entities and relationships in the conceptual schema $C_{1}$ are defined in terms of entities and relationships in $C_{2}$ and $C_{3}$, and these in turn are defined in terms of entities and relationships of $C_{4}$. This allows for the situation that in $C_{1}$ and in $C_{4}$ integrity constraints may be defined on the same entities and relationships. It is important to avoid contradictory integrity constraints in the schema definition, and if all four database systems were of the cooperative type, this would be no problem. If in $C_{1}$ a new integrity constraint is to be defined, then $\mathrm{DS}_{1}$ can communicate with the underlying database systems to determine whether this new constraint violates an already existing constraint. If so, this constraint will be rejected from the definition of $C_{1}$; otherwise it will be accepted. Hence, it is imperative that cooperative database systems contain a constraint validator, if the schema architecture is allowed to be an arbitrary graph.

In the noncooperative case information contained in the data dictionary cannot be exchanged, and therefore checking whether a new constraint violates existing constraints is not possible. A cyclic schema may lead to a violation of integrity constraints. In order to avoid the definition of an inconsistent constraint, only a strict hierarchy of schemas is allowable. 


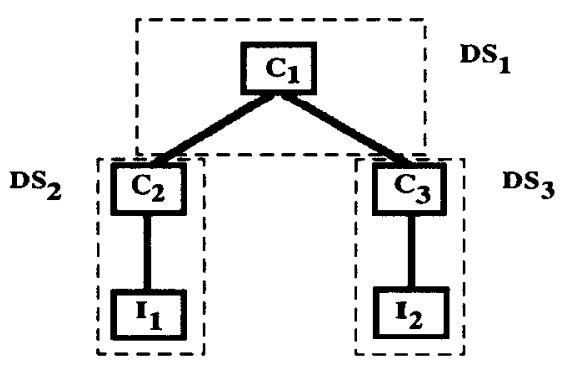

Fig. 18. Virtual and physical database systems.

\subsection{REQUIREMENTS ON COOPERATIVENESS FOR TRANSACTION PROCESSING}

As mentioned before, most research on distributed database systems has concentrated on homogeneous systems and not on heterogeneous ones. In this section we will discuss the functionality of heterogeneous systems and concentrate especially on the implications imposed on query processing and concurrency control by the type of communication.

Let us consider the configuration depicted in Figure 18. Assume that $\mathrm{DS}_{2}$ and $\mathrm{DS}_{3}$ are two noncooperative database systems over which we want to define another database having conceptual schema $C_{1}$. The latter is supported by $\mathrm{DS}_{1} . C_{1}$ will be called a virtual database because it does not have data of its own, and $\mathrm{DS}_{1}$ will correspondingly be called a virtual database system. On the other hand, $C_{2}$ and $C_{3}$ are physical databases, and $\mathrm{DS}_{2}$ and $\mathrm{DS}_{3}$ are physical database systems.

Let us discuss now the scenario for query optimization and processing when a query is issued to $\mathrm{DS}_{1}$ and when DS $\mathrm{DS}_{2}$ and $\mathrm{DS}_{3}$ are noncooperative systems. In this case, $\mathrm{DS}_{1}$ will translate the request according to the mappings $C_{1}-C_{2}$ and $C_{1}-C_{3}$ into two subqueries, to be processed by $\mathrm{DS}_{2}$ and $\mathrm{DS}_{3}$. The subqueries are sent to the physical database systems, where they are processed, and the answers are returned to the virtual database system, where integration of these results will be done. So during query processing no data transmissions between the physical database systems will take place.

In the cooperative case the query-processing costs can be reduced substantially if additional information such as data distribution, access paths, and various statistics is provided for the mappings $C_{1}-C_{2}$ and $C_{1}-C_{3}$. If all this information is given to the query optimizer at the top of the hierarchy, i.e. to the optimizer of the virtual database system, it will be able to perform some global query optimization and compute a much more efficient schedule than if the original query is merely decomposed into subqueries which are optimized 
locally by the independent query optimizers of the physical database systems, which do not cooperate. If, in addition, the transmission of data between the physical databases is allowed, then the query optimizer of the virtual database system can consider an even larger class of schedules to obtain a more efficient solution. The schedule corresponding to the solution will consist now of operations on the access paths and of data transmissions.

In summary, the requirements for query optimization and processing in a cooperative environment are:

(1) the capability to interrogate the data dictionary of another database system and to process the answers by the query optimizer;

(2) the capability to receive data from another database system, to perform a local operation on it, and to transmit the result to another database system.

In our previous discussion we assumed that all cooperative database systems can communicate at the query level. However, it also is sometimes necessary to integrate into a cooperative environment a database system that originally does not have a query-language capability. The question of what constitutes an adequate subset of queries to interrogate the data dictionary of such a system remains open for research.

Before looking at the restrictions on concurrency control imposed by the type of communication used, we will introduce some terminology. Transactions issued to a virtual database system are called global transactions. The resulting transactions for the underlying database systems are called subtransactions. Transactions issued directly to the underlying database systems are called local transactions. Note: a local transaction on one level might be a global transaction on another level. In the following discussion we will assume the configuration of Figure 18.

In the noncooperative case the virtual database only can issue subtransactions to the underlying database systems. These subtransactions are executed independently by the underlying physical database systems, each having its own concurrency control mechanism. The limitations in this type of environment are [2]:

(1) it is not possible to prohibit local transactions from seeing updates of subtransactions;

(2) concurrency control among global transactions cannot have a level of granularity smaller than an entire physical database.

In [2] two proposals for concurrency control are discussed: one is based on sagas, and the other is called altruistic locking. Both are discussed briefly here.

Sagas are intended for applications where it is not necessary to serialize the global transactions. A saga is a global transaction that can be broken up into a collection of subtransactions that can be interleaved arbitrarily with other 
transactions. The subtransactions of a saga are executed as a nonatomic unit. The only guarantee that the virtual database system makes is that of semantic atomicity: if a saga is aborted, any subtransactions in the saga that have already been committed will be undone by compensating subtransactions. This means that compensating subtransactions should be provided for each subtransaction.

A second approach is based on altruistic locking, an extension of two-phase locking. Altruistic locking, like two-phase locking, produces serializable schedules for global transactions if the local concurrency control mechanisms guarantee serializable schedules and the level of locking granularity is an entire physical database. Altruistic locking, however, potentially allows for greater concurrency than two-phase locking, in the sense that the global transactions that can be executed under two-phase locking are a subset of those that can be executed under altruistic locking. In order to guarantee serializable schedules for all transactions, including the local transactions, an additional constraint to be observed is that only one subtransaction per physical database system can be generated by a global transaction. We observe here that this constraint, originally introduced in [9], is present in all approaches which guarantee serializability, whether in a cooperative or in a noncooperative environment.

The basic idea behind altruistic locking is that if a global transaction does not need to access an underlying physical database any further, it may release its lock on that database. The databases released by transactions that are still executing may be accessed by other global transactions under certain conditions. Under the simplest protocol, a global transaction either must not lock any databases which have been released by any global transaction that is still executing, or, alternatively, the released databases that it locks must all have been released by one global transaction that is still active.

In the cooperative case the underlying physical databases may exchange information about the status of the local transactions. Various approaches are foreseeable, depending upon the type of information that can be exchanged and the level of autonomy that is allowed.

In [19] a scheme that is based on the physical databases exchanging order elements (timestamps) with the virtual database system is presented. It is shown that the various existing concurrency control mechanisms can provide these order elements with few or no changes to the algorithms. For example, for two-phase locking protocols, the time at which a subtransaction released its first lock can be used as its order element. Order elements for subtransactions are combined into an order vector for a global transaction. The concurrency control provided by the virtual database system is based on an optimistic approach. The physical database systems where the subtransactions of a global transaction are executed return an order element at the moment they are 
ready to commit. The virtual database system combines the order elements into an order vector and verifies if this vector can find its place in the total ordering of all committed global transactions. The virtual database system functions as the coordinator for the commit protocol, which means that the underlying database systems cannot independently commit the subtransactions.

In [8] an extension to the above scheme is proposed in which it is assumed that the virtual database system knows enough about the behavior of the subtransactions so that it can record in advance their read and write sets. As above, the virtual database system checks for serializability and coordinates the commit protocol. The basic difference from [19] is that the order vector of a global transaction attempting to commit is checked for insertion into the total ordering only if there is a conflict between its read/write sets and those of the recently committed global transactions. The feasibility of these approaches based on order vectors requires the existence of a fast method to check conflicts in ordering with already committed transactions.

\section{CONCLUSION}

We have characterized the basic frameworks for schema architecture which can be supported by distributed database systems and multidatabase systems and have shown how an arbitrary schema architecture can be mapped to a number of physical processors. Since logical integration seems the alternative most promising for the future, we discussed the issues encountered in this approach in multidatabase systems and how an object-oriented approach based on modules can provide an appropriate basis for specifying the mapping information in an arbitrary schema hierarchy.

We introduced the notions of cooperativeness and noncooperativeness of database systems to describe the level of communication permissible among them. Then we discussed in some detail the type of information required to support efficient query processing and concurrency control in a cooperative environment. Furthermore, we have shown that the type of communication possible has further implications on the schema architecture. Thus, to support the enforcement of integrity constraints in a noncooperative environment, we limit ourselves to a strict hierarchy of schemas, while to allow for global query optimization in a cooperative environment, the mapping information must be augmented dynamically with information in the data directory.

We would like to thank Amon Rosenthal of Computer Corporation of America for his helpful comments on an earlier version of this paper. 


\section{REFERENCES}

1. M. Adiba, J. C. Chupin, R. Demolombe, G. Gardarin, and J. Le Bihan, Issues in distributed data base management systems: A technical overview, in Proceedings of the 3rd International Conference on Very Large Databases, West Berlin, 1978, pp. 89-110.

2. R. Alonso, II. Garcia-Molina, and K. Salem, Concurrency control and recovery for global procedures in federated database systems, Data Engrg. 10(3):5-11 (1987).

3. P. Apers, A. Hevner, and S. B. Yao, Optimization algorithms for distributed queries, IEEE Trans. Software Engrg. SE-1(1):57-68 (1983).

4. P. Apers, Data Allocation in Distributed Database Systems, Technical Report, Twente Univ. of Technology, 1984; ACM Trans. Database Systems, to appear.

5. S. Ceri and G. Pelagatti, Distributed Databases: Principles \& Systems, McGraw-Hill, 1984.

6. U. Dayal and H. Y. Hwang, View definition and generalization for database integration in a multidatabase system, IEEE Trans. Software Engrg. SE-10(6):528-644 (Nov. 1984).

7. R. El-Masri and S. Navathe, Object integration in logical database design, in Proceedings of the IEEE COMPDEC Conference, Anaheim, Calif., 1984, pp. 426-433.

8. A. Elmagarmid and Y. Leu, An optimistic concurrency control algorithm for heterogeneous distributed database systems, Data Engrg. 10(3):26-32 (1987).

9. V. Gligor and R. Popescu-Zeletin, Transaction management in distributed heterogeneous database management systems, Inform. Systems 11, No. 4 (1986).

10. J. Gray, The transaction concept: Virtues and limitations, in Proceedings of the 7 th International Conference on Very Large Databases, Cannes, France, 1981, pp. 144-159.

11. D. Heimbigner and D. McL end, A federated architecture for information management, ACM Trans. Office Inform. Systems 3(3):253-278 (1985).

12. H. Y. Hwang, U. Dayal, and M. Gouda, Using semiouterjoins to process queries in multidatabase systems, in Proceedings of the Third ACM SIGACT-SIGMOD Symposium on Principles of Database Systems, Waterloo, Canada, Apr. 1984, pp. 153-162.

13. T. Landers and R. L. Rosenberg, An overview of Multibase, in Distributed Databases, (H. J. Schneider, Ed.), North-Holland, 1982, pp. 153-184.

14. W. Litwin et al., siRius system for distributed data management, in Distributed Data Bases, (H. J. Schneider, Ed.), North-Holland, 1982, pp. 311-366.

15. W. Litwin and K. Kabbaj, Multidatabase interoperability, Computer 19(12):10-18 (1986).

16. L. Mark and N. Roussopoulos, The new database architecture framework-a progress report, in Proceedings of the IFIP WG 8.1 Working Conference on Theoretical and Formal Aspects of Information Systems, North Holland, 1986.

17. A. Motro, Superviews: Virtual integration of multiple databases, IEEE Trans. Software Engrg. SE-13(7):785-798 (1987).

18. E. J. Neuhold and B. Walter, An overview of the architecture of the distributed data base system POREL, in Distributed Databases (H. J. Schneider, Ed.), North-Holland, 1982, pp. 247-290.

19. C. Pu, Superdatabases: Transactions across database boundaries, Database Engrg. 10(3):19-24 (1987).

20. J. B. Rothie, P. Bernstein, S. Fox, N. Goodman, M. Hammer, T. Landers, C. Reeve, D. Shipman, and E. Wong, Introduction to a system for distributed databases (SDD-1), ACM Trans. Database Systems 5(1):1-17 (1980).

21. P. Scheuermann, G. Schiffner, and $\mathrm{H}$. Weber, Abstraction capabilities and invariant properties modeling within the entity-relationship approach, in Entity-Relationship Approach to System Analysis and Design (P. Chen, Ed.), North Holland, 1980, pp. 121-140. 
22. G. Schiffner, P. Scheuermann, S. Seehusen, and H. Weber, On a specification and performance evaluation model for multicomputer database machines, in Proceedings of the Third International Workshop on Database Machines, Springer-Verlag, Munich, 1983, pp. 46-73.

23. V. Schiel, A. Furtado, E. Neuhold, and M. Casanova, Towards multi-level and modular conceptual schema specifications, Inform. Systems 9(1):43-57 (1984).

24. M. Stonebraker and E. Neuhold, A distributed database version of INGREs, in Proceedings of the 2nd Berkeley Workshop on Distributed Data Management and Computer Networks, 1977, pp. 19-38.

25. M. Templeton, D. Bull, A. Hwang, I. Kameny, and E. Lund, An overview of the MERMAID System: A frontend to heterogeneous databases," in Proceedings of the IEEE EASCON 1983 Conference, Washington, 1983, pp. 387-402.

26. D. Tsichritzis and A. Klug (Eds.), The ANSI/X3/SPARC DBMS framework, Inform. Systems 3, No. 3 (1978).

27. H. Weber, Object-oriented DDBMS-Design, in Proceedings of the 2nd ICOD Conference, 1983, pp. 136-221.

28. $\mathrm{H}$. Weber and $\mathrm{H}$. Ehrig, Specification of modular systems, IEEE Trans. Software Engrg. SE-12(7):784-798 (1986).

29. $\mathrm{R}$. Williams et al., $\mathrm{R}^{*}$ : An overview of the architecture, in Improving Database Usability and Responsiveness, (P. Scheuermann, Ed.), Academic, 1982, pp. 1-28.

Received 12 July 1987; revised 10 January 1988 